\title{
Structural and functional characterization of a novel cold-active $S$-formylglutathione hydrolase (SfSFGH) homolog from Shewanella frigidimarina, a psychrophilic bacterium
}

Chang Woo Lee ${ }^{1 \dagger}$, Wanki Yoo ${ }^{2,3 \dagger}$, Sun-Ha Park ${ }^{1,6 \dagger}$, Ly Thi Huong Luu Le ${ }^{2}$, Chang-Sook Jeong 1,4 , Bum Han Ryu ${ }^{2,3}$, Seung Chul Shin ${ }^{1}$, Han-Woo Kim ${ }^{1,4}$, Hyun Park ${ }^{5}$ Kyeong Kyu Kim ${ }^{3}$ T. Doohun Kim² and Jun Hyuck Lee ${ }^{1,4^{*}}$

\begin{abstract}
Background: S-Formylglutathione is hydrolyzed to glutathione and formate by an S-formylglutathione hydrolase (SFGH) (3.1.2.12). This thiol esterase belongs to the esterase family and is also known as esterase D. SFGHs contain highly conserved active residues of Ser-Asp-His as a catalytic triad at the active site. Characterization and investigation of SFGH from Antarctic organisms at the molecular level is needed for industrial use through protein engineering.

Results: A novel cold-active S-formylglutathione hydrolase (SFSFGH) from Shewanella frigidimarina, composed of 279 amino acids with a molecular mass of $\sim 31.0 \mathrm{kDa}$, was characterized. Sequence analysis of SFSFGH revealed a conserved pentapeptide of G-X-S-X-G found in various lipolytic enzymes along with a putative catalytic triad of Ser148Asp224-His257. Activity analysis showed that SfSFGH was active towards short-chain esters, such as $p$-nitrophenyl acetate, butyrate, hexanoate, and octanoate. The optimum pH for enzymatic activity was slightly alkaline (pH 8.0). To investigate the active site configuration of SfSFGH, we determined the crystal structure of SfSFGH at $2.32 \AA$ resolution. Structural analysis shows that a Trp182 residue is located at the active site entrance, allowing it to act as a gatekeeper residue to control substrate binding to SFSFGH. Moreover, SFSFGH displayed more than $50 \%$ of its initial activity in the presence of various chemicals, including 30\% EtOH, 1\% Triton X-100, 1\% SDS, and $5 \mathrm{M}$ urea.

Conclusions: Mutation of Trp182 to Ala allowed SfSFGH to accommodate a longer chain of substrates. It is thought that the W182A mutation increases the substrate-binding pocket and decreases the steric effect for larger substrates in SFSFGH. Consequently, the W182A mutant has a broader substrate specificity compared to wild-type SFSFGH. Taken together, this study provides useful structure-function data of a SFGH family member and may inform protein engineering strategies for industrial applications of SFSFGH.
\end{abstract}

Keywords: Crystal structure, S-Formylglutathione hydrolase, Substrate specificity, Shewanella frigidimarina, Mutagenesis

\footnotetext{
*Correspondence: doohunkim@sookmyung.ac.kr;

junhyucklee@kopri.re.kr

${ }^{\dagger}$ Chang Woo Lee, Wanki Yoo and Sun-Ha Park contributed equally to this

work

${ }^{1}$ Unit of Polar Genomics, Korea Polar Research Institute, Incheon 21990,

Republic of Korea

${ }^{2}$ Department of Chemistry, College of Natural Science, Sookmyung

Woman's University, Seoul 04310, Republic of Korea

Full list of author information is available at the end of the article
} 


\section{Background}

Esterases hydrolyze ester bonds and produce cleaved acid and alcohol compounds. Although they have low sequence identity and diverse substrate specificities as well as biological functions, most esterases adopt a common $\alpha / \beta$ hydrolase fold and contain a highly conserved serine-histidine-aspartic acid catalytic triad at the active site [1-3]. $S$-formylglutathione hydrolase (SFGH, EC 3.1.2.12) is a member of the esterase family and is also known as esterase D. SFGH hydrolyzes $S$-formylglutathione into glutathione and formic acid and can be broadly considered an esterase, or more specifically, a thioesterase. This enzyme is involved in the glutathionedependent formaldehyde detoxification pathway, which prevents the negative effects of formaldehyde by a series of enzymatic reactions involving formaldehyde dehydrogenase (FALDH, S-hydroxymethylglutathione dehydrogenase) and SFGH, which depend upon the spontaneous binding of glutathione to formaldehyde. Indeed, the expression levels of FALDH (frmA) and SFGH (frmB) in Escherichia coli cells are remarkably increased by 20to 100-fold over basal levels when subjected to formaldehyde treatment [4]. Notably, a double-mutant strain of $E$. coli with the deletion of both frmB and paralogous yeiG genes, which all encode SFGH, showed a remarkably reduced growth rate in the presence of formaldehyde [5]. Likewise, a mutant strain of Paracoccus denitrificans for the SFGH gene $(f g h A)$ was unable to grow on methanol or methylamine, which resulted in the formation of formaldehyde [6]. Considering the importance of this pathway, SFGH genes are widely conserved in diverse organisms, including prokaryotes and eukaryotes. In humans, esterase $\mathrm{D}$, which was subsequently proven to be identical to SFGH, has been identified, characterized, and structurally determined. This enzyme has attracted much attention as its polymorphisms are related to several diseases, including retinoblastoma and Wilson's disease [7-9]. In addition, SFGHs were found in other eukaryotes including plants (Arabidopsis thaliana $[10,11]$ and Pisum sativum [12]) and yeast (e.g., Saccharomyces cerevisiae [13] and Candida boidinii [14]). Bacterial SFGHs were also identified in various species including Paracoccus denitrificans [6], Agrobacterium tumefaciens [15], and E. coli [5].

As mentioned above, many studies on SFGHs have been conducted, which have revealed the features of SFGHs. Sequence alignment analyses have shown that SFGH has a conserved sequence motif (GHSMGG) that harbors catalytic serine; thus, this enzyme is also referred to as a serine hydrolase. This serine residue, along with the conserved aspartate and histidine residues, forms a catalytic triad of SFGH. Two conserved residues of leucine and methionine that form an oxyanion hole are also found in close proximity to the catalytic serine.
Furthermore, enzymes belonging to the SFGH class generally contain five large blocks of conserved residues [5]. Functional characterization studies demonstrated that SFGH could also exhibit carboxyl esterase activity towards xenobiotic esters (e.g., methylumbelliferyl acetate) in addition to having thioesterase activity [10, 11, 13]. Many SFGHs are known to be active towards $p$-nitrophenyl esters or naphthyl ester derivatives as well as formyl glutathione.

In this study, we characterized a novel cold-active $S$-formylglutathione hydrolase (SfSFGH) homolog from a psychrophilic bacterium, Shewanella frigidimarina. Shewanella frigidimarina is a facultative anaerobic Gram-negative bacterium with rod-like shape, originally isolated from Antarctic marine environments. To date, only two studies have characterized SFGH from Arctic/ Antarctic organisms: OLEI01171 from Oleispira antarctica [16] and PhEST from Pseudoalteromonas haloplanktis $[17,18]$. However, the structural and functional properties of $S$-formylglutathione hydrolase from psychrophiles are still poorly understood. In this context, our study will enrich the pool of SFGH, providing useful information regarding the sequence, biophysical, and enzymatic characteristics of SFGH. Furthermore, the crystal structure of SfSFGH has been determined at $2.32 \AA$ resolution. Several substrate-binding site residue mutants have been generated to alter the substrate specificity and flexibility of SfSFGH. Interestingly, the W182A mutant showed remarkably increased substrate flexibility compared to wild-type SfSFGH. Collectively, these findings provide useful insights to inform the protein engineering of bacterial esterases as industrially useful biocatalysts.

\section{Results and discussion}

\section{Biochemical characterization of SFSFGH}

The sequence of SfSFGH (GenBank I.D.: ABI73260.1) was previously annotated as a carboxylesterase with little additional information. Sequence alignment of SfSFGH with similar sequences retrieved from PSI-BLAST indicates that these enzymes share highly conserved sequences, including a 'GHSMGG' motif. These enzymes are now classified as a PF00756 esterase family based on sequence homology [15, 19-21]. As the 'GHSMGG' motif is a representative feature of the esterase/lipase family $\mathrm{V}$, a phylogenetic tree was generated containing SfSFGH with other enzymes belonging to the esterase/lipase family from I to VIII. As expected, SfSFGH was classified into the esterase/lipase family $\mathrm{V}$ and showed a clear separation from other families (Additional file 1: Fig. S1). Furthermore, it is generally known that the 'PAL' motif is one of the representative features of the esterase/lipase family V [22]. Together with SfSFGH, other PF00756 esterases, 
such as OLEI01171 and PhEst, have the 'PAL' and 'PML' motifs, respectively. Collectively, sequence analysis data suggest that SfSFGH might be a new member of the esterase/lipase family V. In addition, SfSFGH shows significant sequence homology with other bacterial SFGHs (66.43\% sequence identity with Pseudoalteromonas haloplanktis TAC125 SFGH, 54.48\% with Paracoccus denitrificans SFGH, 50.91\% with Agrobacterium tumefaciens SFGH, and $67.93 \%$ with $E$. coli $\mathrm{SFGH}$ ). We also performed gene cluster analysis of SfSFGH, and found that gene clusters surrounding the SFGH gene were highly conserved in Shewanella species, including S. sediminis, S. woodyi, S. pealeana, and S. piezotolerans (Additional file 1: Fig. S2).

Recombinant SfSFGH protein was expressed in E. coli and purified by His-tag affinity chromatography and size-exclusion chromatography (Additional file 1: Fig. S3). Analytical ultracentrifugation (AUC) analysis with SfSFGH showed a dimer mass of $62.3 \mathrm{kDa}$ (sedimentation coefficient, $4.209 \mathrm{~S}$; frictional ratio, 1.34). The substrate specificity of recombinant SfSFGH protein was determined using $p$-nitrophenyl esters with different acyl-chain lengths. As shown in Fig. 1a, SfSFGH showed a preference toward short-chain substrates, including $p$-NA, $p-\mathrm{NB}, p-\mathrm{NH}$, and $p-\mathrm{NH}$, with more than $50 \%$ of its maximum activity. However, less than $15 \%$ of relative enzymatic activities were detected towards longer-chain substrates, such as $p$-ND and $p$-NDD, and almost no enzymatic activity was observed with $p$-NP. The optimum $\mathrm{pH}$ for SfSFGH was determined to be in the range of $\mathrm{pH}$ 3.0 to 10.0 (Fig. 1b). SfSFGH showed maximum activity at $\mathrm{pH} 8.0$ and $\sim 40 \%$ activity at $\mathrm{pH} 7.0$. In contrast, SfSFGH exhibited almost no enzymatic activity in other $\mathrm{pH}$ ranges. The kinetic parameters of SfSFGH were determined using $p$-NA. SfSFGH exhibited typical Michaelis-Menten kinetics with a hyperbolic plot of reaction velocity as a function of substrate concentration. Based on the Lineweaver-Burk plot with linear regression, the
Michaelis-Menten constant $\left(K_{\mathrm{m}}\right)$ and maximum velocity $\left(V_{\max }\right)$ were $515 \mu \mathrm{M}( \pm 42.8)$ and $0.319 \mu \mathrm{M} \mathrm{s}^{-1}( \pm 0.0254)$, respectively (Fig. 1c). The calculated $k_{\text {cat }}$ of SfSFGH was $0.310 \mathrm{~s}^{-1}( \pm 0.0258)$, which is almost six times less than that of AtuSFGH and PhEST $\left(1.95 \mathrm{~s}^{-1}\right.$ and $2 \mathrm{~s}^{-1}$, respectively) $[15,17]$. Notably, enzymatic activity assay results for several different temperatures showed that SfSFGH has high activity at low temperature $\left(4{ }^{\circ} \mathrm{C}\right)$, but starts to lose activity at $\sim 37^{\circ} \mathrm{C}$ (Fig. 2).

\section{Overall structure of SfSFGH}

The crystal structure of SfSFGH was determined to a resolution of $2.32 \AA$ using a molecular replacement method (Fig. 3). The crystal structure of esterase (OLEI01171) from Oleispira antarctica (sequence identities 83\%; PDB cod 3i6y) was used as a template model [16]. SfSFGH was crystallized in space group $P 2_{1}$ with two monomers in the asymmetric unit. The final model contained 208 water molecules and 554 amino acids. The monomeric structure of SfSFGH includes $12 \alpha$-helices and $9 \beta$-strands (Fig. 3a). The $\beta 1-\beta 2-\beta 3$ strands form an antiparallel $\beta$-sheet, and the $\beta 4$ to $\beta 9$ strands form a parallel $\beta$-sheet. These two $\beta$-sheets are linked and assemble into a large central $\beta$-sheet. The $12 \alpha$-helices surround both sides of the large $\beta$-sheet. These features of the overall architecture show considerable similarity with those of the previously reported $S$-formylglutathione hydrolases (SFGHs). Structural alignments using a Dali search showed that the esterase (OLEI01171) from Oleispira Antarctica (PDB code 3i6y) has the highest Z-score with 50.5 [16, 23]. Crystal structures of $S$-formylglutathione hydrolase from Pseudoalteromonas haloplanktis (PDB code 3ls2), esterase D from Neisseria meningitidis (PDB code 4b6g), and $S$-formylglutathione hydrolase from Agrobacterium fabrum (PDB code 3e4d) also showed high structural similarities (Table 1) $[15,18,24]$.

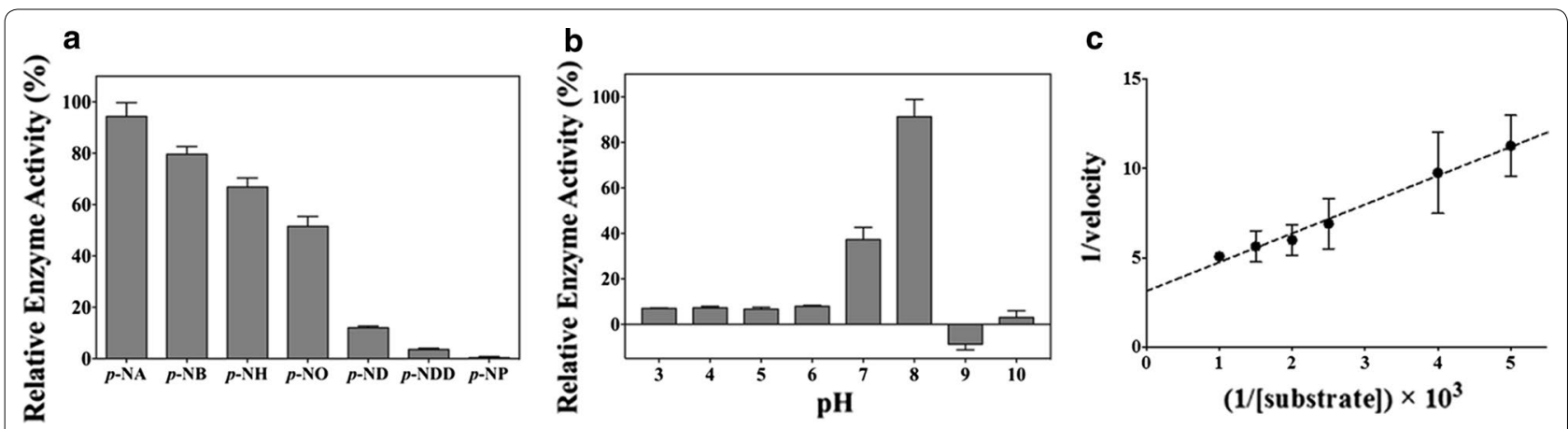

Fig. 1 Enzymatic activity of SfSFGH. a Substrate specificity was investigated using $p$-nitrophenyl esters with different acyl-chain lengths. $\mathbf{b}$ Effects of $\mathrm{pH}$ on enzymatic activity were studied from pH 3.0 to 10.0. Activity at the optimal pH was set as $100 \%$. c Lineweaver-Burk plots showing the reciprocal of the velocity of SfSFGH versus the reciprocal of the substrate concentration. All experiments were performed in triplicate 

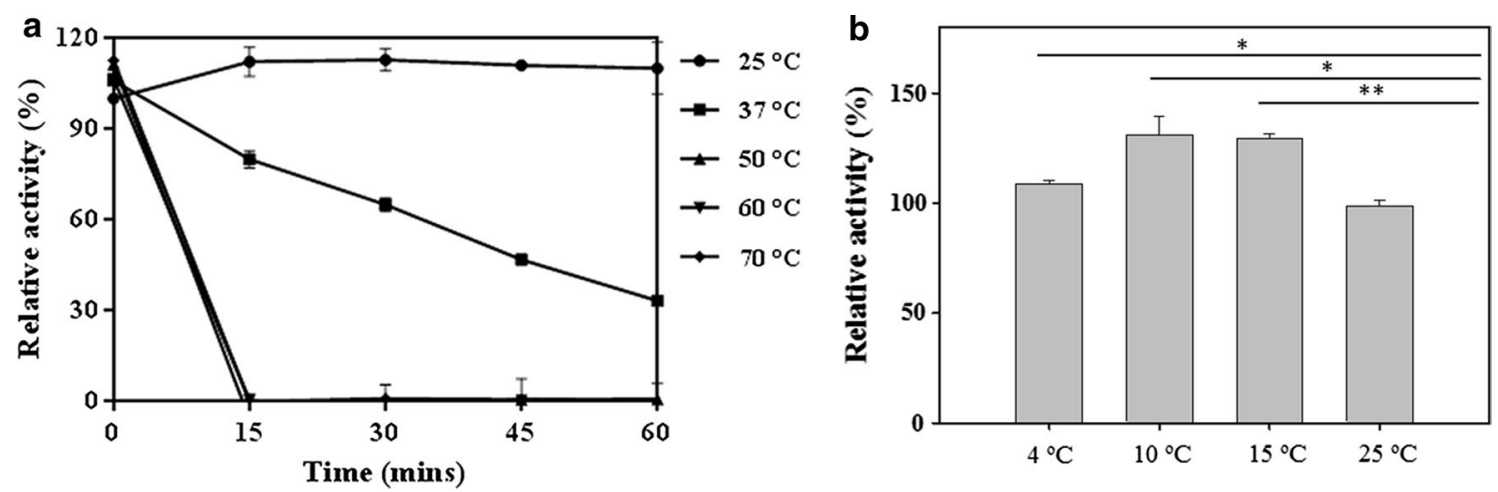

Fig. 2 Effects of temperature on the activity of SfSFGH. a Thermal stability of SfSFGH. SfSFGH was heated at various temperatures, ranging from 25 to $70^{\circ} \mathrm{C}$. Then, $10 \mathrm{\mu g}$ SfSFGH was collected every $15 \mathrm{~min}$ and the residual activity was measured using $p$-NA as a substrate. Activity is shown relative to the initial activity. $\mathbf{b}$ Enzymatic activity of SfSFGH was evaluated at different temperatures. Relative activity of SfSFGH was measured using $p$ NP- $C_{2}$ as a substrate after $1 \mathrm{~h}$ incubation at each temperature. A two-tailed unpaired Student $t$ test was used to evaluate statistical significance: ${ }^{*} P<0.05$ and ${ }^{* *} P<0.005$. All experiments were performed in triplicate

\section{Active site of SfSFGH}

The putative substrate-binding site of SfSFGH is located at the end of the $\beta 6$-strand. Including the $\alpha 2, \alpha 8$, and $\alpha 12$ helices, the $\beta 4-\alpha 2$ loop, $\beta 8-\alpha 11$ loop, and $\beta 9-\alpha 12$ loop regions together form a groove architecture. The conserved catalytic triad residues of Ser148, Asp224, and His 257 are located in this groove (Fig. 4). The catalytic residue Ser148 is located in the short $\beta 6-\alpha 6$ loop region and directly interacts with His 257 located in the $\beta 9-\alpha 12$ loop region. Similarly, the residue Asp224 located on the $\beta 8-\alpha 11$ loop region interacts with His257. In addition, the oxyanion hole comprising the backbone amides Leu55 and Met149 can be observed at the neighboring active site. In the apo state of the SfSFGH structure, oxyanion holes are occupied by water molecules. The vicinity of the substrate-binding site is positively charged by the Lys65 and His147. This positively charged area might induce binding of an acidic substrate, such as an acyl group. Moreover, the residues Cys57, Asn61, Trp182, and Phe226 are conserved in the substrate-binding site of SfSFGH. Especially in SFGH from Saccharomyces cerevisiae, the W197I mutant (Trp182 of SfSFGH) showed highly increased substrate affinities [25]. Likewise, mutation of Trp182 to alanine in SfSFGH affected its activity. Detailed data for the W182A mutant are provided in the next section.

\section{Site-directed mutagenesis to increase the substrate-binding pocket size of SFSFGH}

Based on this structural information, we hypothesized that remodeling the substrate-binding pockets of SfSFGH would allow the enzyme to exhibit broadened substrate specificity. Therefore, several mutants targeting the substrate-binding pocket residues (L55A, L55V, H147A, W182A, and F226A) were designed, constructed, purified, and characterized with respect to their activity (Fig. 5a). Interestingly, the W182A mutant showed substantially increased activity for large and bulky substrates compared to wild-type SfSFGH. Notably, the W182A mutant showed a substantially altered preference for longer substrates ( $p$-NB and $p$-NH), unlike wild-type SfSFGH (Fig. 5a, b). Likewise, the $K_{m}$ values of W182A for $p$-NA, $p$-NB, and $p$-NO were $1.393( \pm 0.136), 0.303$ $( \pm 0.049)$, and $0.459( \pm 0.085)$, respectively. The calculated catalytic efficiency $\left(k_{c a t} / K_{m}\right)$ of W182A for $p$-NB $\left(26.4 \mathrm{~s}^{-1} \mathrm{mM}^{-1}\right)$ was 20 times greater than that for $p$-NA

\footnotetext{
(See figure on next page.)

Fig. 3 Crystal structure of SFSFGH. a The overall structure of SFSFGH is shown as a ribbon diagram with a-helices (aquamarine color) and $\beta$-strands (salmon color). The catalytic triad residues (Ser148, Asp24, and His257) are shown as stick models. b Multiple sequence alignments of SfSFGH (NCBI reference sequence number WP_011638859.1) with homologous models. The sequences of esterases from the oil-degrading bacterium Oleispira antarctica (PDB code 3i6y; UniProtKB code D0VWZ4), S-formylglutathione hydrolase from Pseudoalteromonas haloplanktis (PDB code 3ls2; UniProtKB code Q3IL66), S-formylglutathione hydrolase from Agrobacterium tumefaciens (PDB code 3e4d; UniProtKB code A9CJ11), esterase D from humans (PDB code 3fCx; UniProtKB code P10768), esterase D from Neisseria meningitides (PDB code 4b6g; UniProtKB code Q9JZ43), and S-formylglutathione hydrolase from Saccharomyces cerevisiae (PDB code 1 pv1; UniProtKB code P40363) were used for alignment. The conserved motif found in SFGHs is boxed in red. The catalytic triad residues indicated with a black triangle are conserved in all models. The gatekeeper residue of tryptophan is indicated with a black circle. An alignment was prepared using the program ClustalX and edited with GeneDoc. Corresponding secondary structures are shown above the sequences
} 


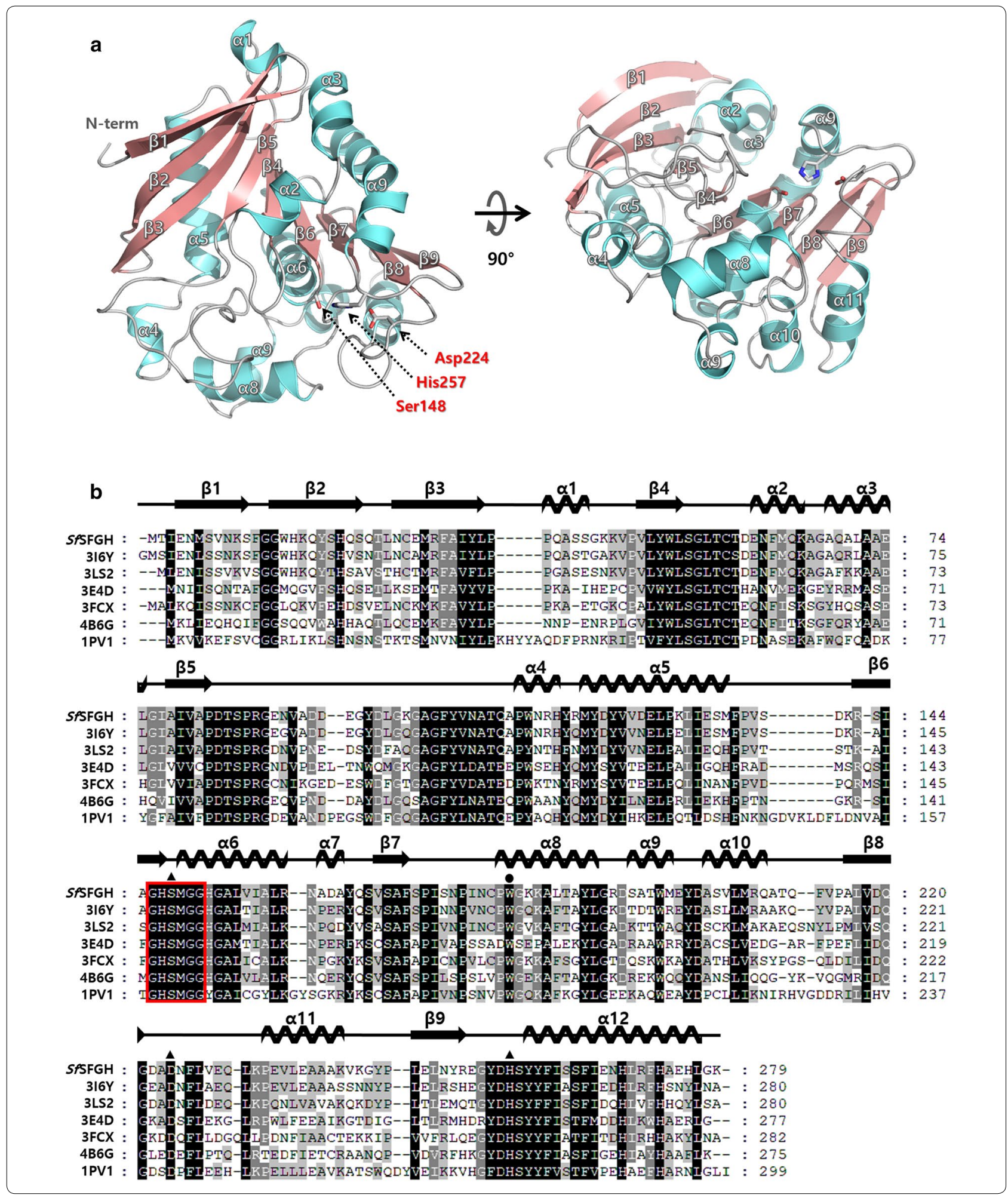

$\left(1.3 \mathrm{~s}^{-1} \mathrm{mM}^{-1}\right)$ (Table 2). In addition, the W182A mutant also showed acetylation activity towards $\alpha$-D-glucose penta-acetate whereas the wild-type did not. It is thought that the W182A mutation probably eliminates the steric hindrance of substrate entry and increases the size of the substrate-binding pocket by removing the bulky side 
Table 1 Selected structural homologues of SfSFGH from a DALI search (DALI-Lite server)

\begin{tabular}{|c|c|c|c|c|c|}
\hline Protein & PDB code & DALI Z-score & R.m.s.d. (Å) & $\begin{array}{l}\text { Sequence \% ID with SfSFGH (aligned } \\
\text { residue number/total residue number) }\end{array}$ & References \\
\hline Esterase APC40077 & $316 Y$ & 50.7 & 0.4 & $81 \%(277 / 278)$ & [16] \\
\hline S-Formylglutathione hydrolase & $3 \mathrm{LS} 2$ & 48.8 & 0.8 & $66 \%(276 / 278)$ & [18] \\
\hline Putative esterase & $4 \mathrm{~B} 6 \mathrm{G}$ & 46.8 & 0.9 & $53 \%(275 / 275)$ & [24] \\
\hline Esterase D & $3 E 4 D$ & 46.4 & 1.0 & $48 \%(276 / 278)$ & [15] \\
\hline S-Formylglutathione hydrolase & $3 F C X$ & 46.0 & 1.0 & $54 \%(271 / 275)$ & [8] \\
\hline $\begin{array}{l}\text { Hypothetical } 33.9 \text { kDa Esterase in } \\
\text { SMC3-MRPL8 }\end{array}$ & 1PV1 & 42.3 & 1.2 & $45 \%(272 / 290)$ & {$[25]$} \\
\hline S-Formylglutathione hydrolase & 4FLM & 42.1 & 1.1 & $44 \%(269 / 288)$ & [38] \\
\hline
\end{tabular}

chain of Trp182 (Fig. 5c, d). However, other mutations were not effective for changing the substrate specificity and improving the activity of SfSFGH. Therefore, the Trp182 residue is important for substrate selectivity and discrimination in SfSFGH. In conclusion, the W182A mutant of SfSFGH has different substrate specificities and unique biochemical characteristics. Thus, the engineered W182A mutant may be useful for biotechnological fields, such as fine chemical synthesis and industrial pharmaceutics.

\section{Structural stability of SfSFGH}

The structural stability of SfSFGH was investigated via thermal and chemical unfolding with circular dichroism and intrinsic fluorescence analyses, respectively. For thermal unfolding, CD signals of SfSFGH were monitored at $222 \mathrm{~nm}$, at temperatures from 20 to $90{ }^{\circ} \mathrm{C}$. In these Far-UV CD measurements, SfSFGH showed a remarkable structural transition at around $45^{\circ} \mathrm{C}$ (Fig. 6a), which is consistent with the results of the enzymatic activity assays (Fig. 2a). In the case of OLEI01171, another coldactive esterase, enzymatic activity decreased when the temperature increased, and the aggregation temperature is similar to that of SfSFGH $\left(\sim 45^{\circ} \mathrm{C}\right)$ [16]. Structural superposition analysis shows how SfSFGH remains catalytically active at low temperatures. SfSFGH has a more open active site compared with mesophilic homologs (human esterase D, PDB code 3FCX, and Saccharomyces cerevisiae SFGH, PDB code 4FLM) [9, 38]. Especially, the $\beta 5$ - $\alpha 4$ loop region (residues $83-112$ ) in SfSFGH has a significantly different conformation with a relatively higher main chain B-factor value $\left(27.9 \AA^{2}\right)$ than overall B-factor value $\left(24.7 \AA^{2}\right.$ ) (Additional file 1: Fig. S4). Our sequence alignment results also show that only cold-active enzymes have a conserved glycine residue in this $\beta 5-\alpha 4$ loop region (Gly96 in SfSFGH and Gly96 in OLEI01171). Collectively, it is thought that the flexibility of the $\beta 5-\alpha 4$ loop region and the open active site are related to the cold-activity of SfSFGH.

Next, chemical unfolding of SfSFGH was monitored based on changes in intrinsic fluorescence with different urea concentrations (from 0 to $5 \mathrm{M}$ ). As shown in Fig. 6b, native SfSFGH exhibited a $\lambda_{\max }$ of $341 \mathrm{~nm}$ in the absence of urea, and a red shift of $\lambda_{\max }$ to $367 \mathrm{~nm}$ was observed in the presence of $5 \mathrm{M}$ urea. Fluorescence intensity was significantly decreased from $1 \mathrm{M}$ urea, and further reduction was nearly proportional to the increase in urea concentration.

In the chemical stability test, SfSFGH showed remarkable residual enzymatic activity in the presence of diverse chemicals (Fig. 6c-e). With organic solvents, SfSFGH could retain $\sim 76 \%, \sim 51 \%$, and $\sim 43 \%$ of its original activity with $10 \% \mathrm{EtOH}, 30 \% \mathrm{EtOH}$, and 30\% I-PrOH, respectively. For detergents, SfSFGH exhibited more than $50 \%$ of its initial activity with $1 \%$ Triton X-100 or SDS up to $1 \%$, although almost no enzymatic activity was observed with $1 \%$ Tween20. Higher SDS concentrations of $2 \%$ and $5 \%$ decreased the enzymatic activity to $30 \%$ and $15 \%$ of its original activity, respectively, rather than inducing complete inhibition of enzymatic activity. Notably, SfSFGH incubated with 5 mM PMSF, a serine hydrolase inhibitor, retained $\sim 36 \%$ of its original activity. To verify the resistance of SfSFGH to hydrogen-bond-disturbing conditions, SfSFGH activity was also examined with urea concentrations ranging from 0 to $5 \mathrm{M}$. The enzymatic activity of SfSFGH was found to be gradually reduced as the concentration of urea increased. Interestingly, SfSFGH retained more than $\sim 50 \%$ of its initial activity at all concentrations. Furthermore, SfSFGH maintained its activity $(\geq 80 \%)$ even after 10 repeated cycles of freezing and thawing (Additional file 1: Fig. S5). Taken together, SfSFGH appears to have notable thermal and chemical stability. Notably, SfSFGH was strongly resistant to organic solvents and detergents such as $\mathrm{EtOH}, \mathrm{i}-\mathrm{PrOH}$, Triton X-100, and SDS, suggesting that it could be useful in industrial applications. 

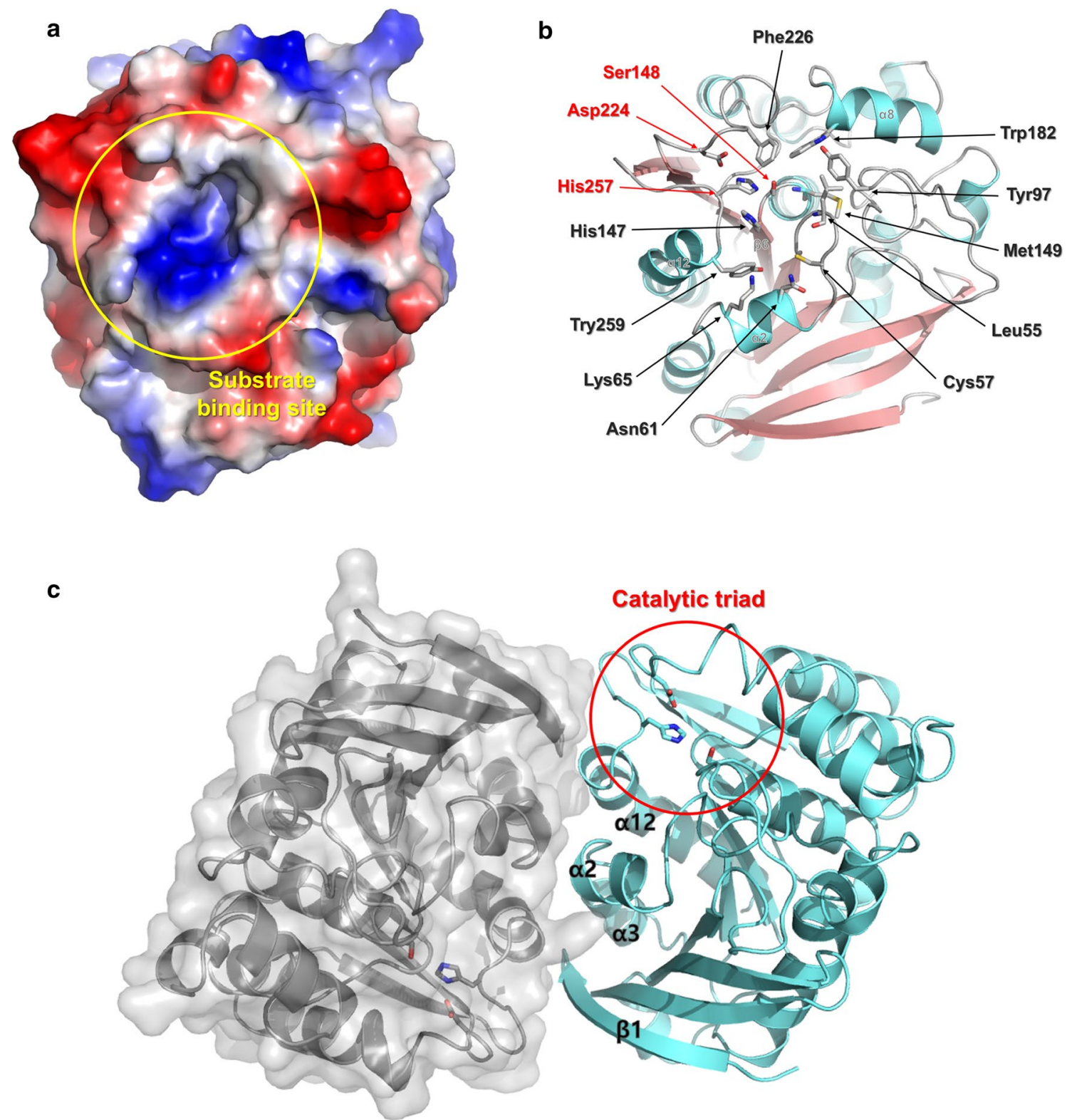

Fig. 4 Active site of SFSFGH. a The substrate-binding site (yellow circle) of SFSFGH has a positively charged surface. b The conserved catalytic triad (Ser148, Asp24, and His257) residues are located on the substrate-binding site. The residues creating a substrate-binding site are presented as a stick model. c Dimerization of SFSFGH is mediated by the $a 2, a 3$, and $a 12$ helices. The $\beta 1$-strand also participates in the dimerization interaction

\section{Conclusion}

In this study, a novel cold-active $S$-formylglutathione hydrolase (SfSFGH) homolog from Shewanella frigidimarina was characterized. Regarding enzymatic activity, SfSFGH was found to prefer short-chain substrates and weakly basic $\mathrm{pH}$ conditions. A single residue mutation (W182A) changed the substrate preference of SfSFGH to longer-chain substrates. This finding provides valuable information for further protein engineering of SfSFGH to obtain more suitable substrate-binding sites. Remarkably,
SfSFGH appears to retain its enzymatic activity in the presence of inhibitory chemicals. These properties of SfSFGH could be very useful in industrial applications that generally require enzymes to show robust activity in harsh conditions.

\section{Materials and methods \\ Protein expression and purification}

Shewanella frigidimarina (KCTC 22881, Korean Collection for Type Cultures (WDCM 597), Seoul, Korea) 


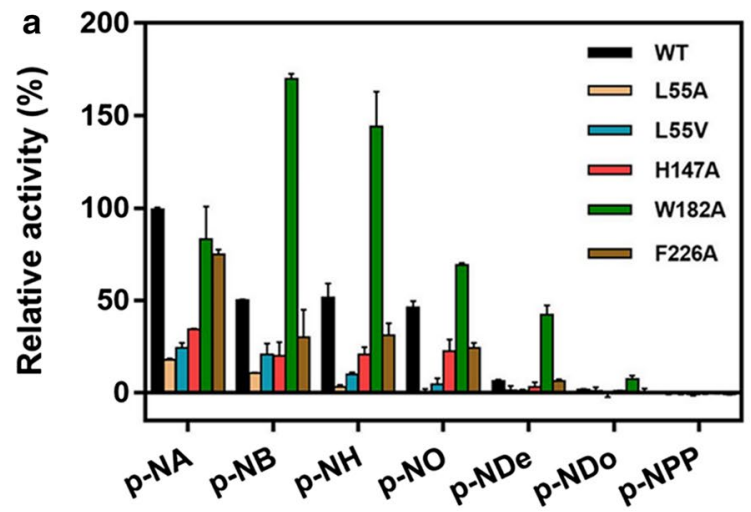

C

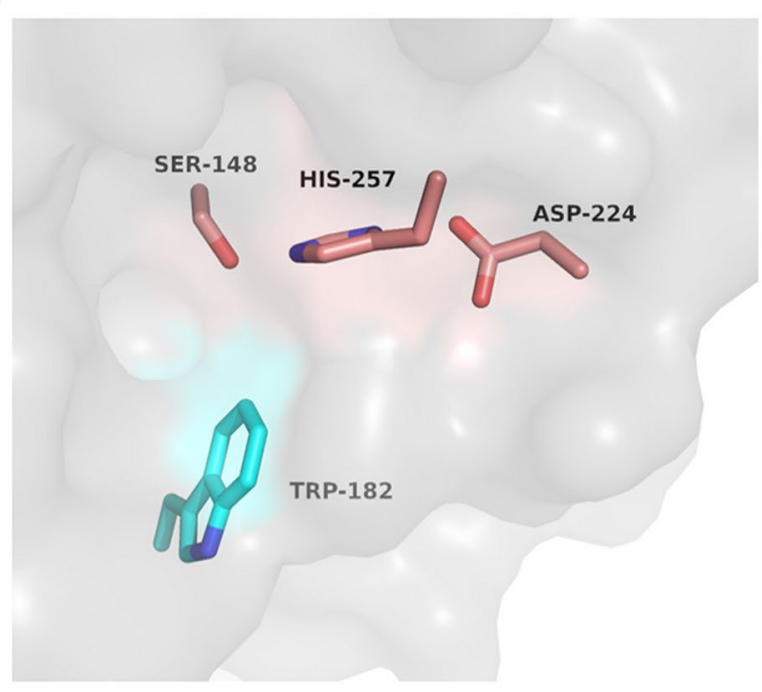

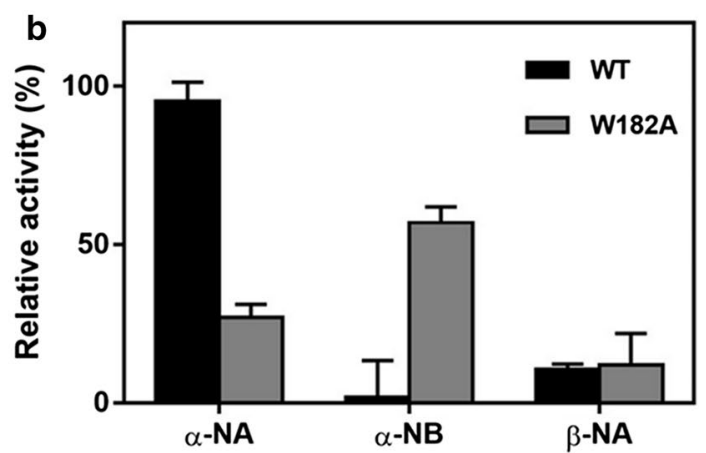

d Carbohydrate assay

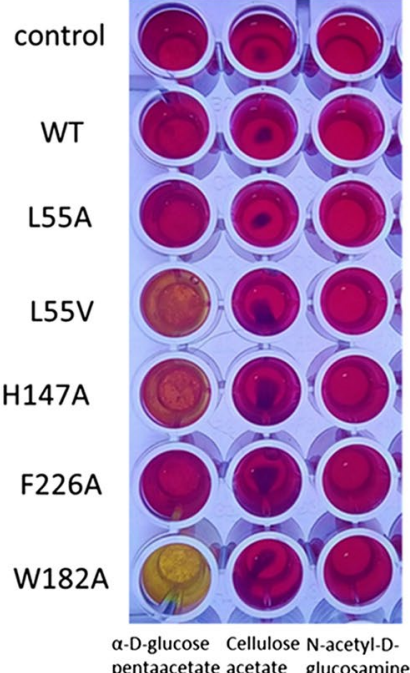

Fig. 5 Effects of mutation on SfSFGH enzymatic activity. a Comparison of relative activities between wild-type SfSFGH and several point mutants (L55A, L55 V, H147A, W182A, and F226A). All mutants are located on the substrate-binding pocket. Assays were carried out using various substrates ( $p$-NA, $p-\mathrm{NB}, p-\mathrm{NH}, p-\mathrm{NO}, p$-NDe, $p$-NDo, and $p$-NPP). The relative activity of wild-type SfSFGH with $p$-NA was set to $100 \%$. $\mathbf{b}$ Increased enzymatic activity for a-NB in the W182A mutant was observed compared with that of wild-type SFSFGH. The relative activity of wild-type SFSFGH with a-NA was set to 100\%. c The surface of the substrate-binding site of SfSFGH is colored grey. The catalytic triad residues (Ser148, Asp224, and His257; salmon) and Trp182 (cyan) are presented as a stick model. $\mathbf{d}$ Hydrolytic activities towards a-D-glucose penta-acetate, cellulose acetate, and $\mathrm{N}$-acetyl-D-glucosamine. All experiments were performed in triplicate

Table $2 K_{m}, k_{\text {cat' }}$ and specificity constant $\left(k_{\text {cat }} / K_{m}\right)$ of the W182A mutant

\begin{tabular}{|c|c|c|c|c|}
\hline Substrates & $\mathrm{V}_{\max }\left(\mu \mathrm{M} \mathrm{s}^{-1}\right)$ & $K_{m}(\mathrm{mM})$ & $k_{\text {cat }}\left(\mathrm{S}^{-1}\right)$ & $\begin{array}{l}k_{\text {cat }} / K_{m} \\
\left(\mathrm{~s}^{-1} \mathrm{mM}^{-1}\right)\end{array}$ \\
\hline$p$-NA & $5.378 \pm 0.268$ & $1.393 \pm 0.136$ & $1.852 \pm 0.092$ & 1.3 \\
\hline$p-\mathrm{NB}$ & $0.861 \pm 0.035$ & $0.303 \pm 0.049$ & $8.003 \pm 0.326$ & 26.4 \\
\hline$p-\mathrm{NO}$ & $0.477 \pm 0.023$ & $0.495 \pm 0.085$ & $0.144 \pm 0.007$ & 0.3 \\
\hline
\end{tabular}

was cultured in marine medium 2216 (BD Difco, USA). Chromosomal DNA was extracted and purified using a DNeasy Tissue and Blood Kit (Qiagen, USA) according to the manufacturer's instructions. The open reading frame of the SfSFGH gene was amplified using polymerase chain reaction with the following primers: forward, 5'-CCTGCTAGCATGACAATTGAAAATATGAG-3'; reverse, 5'-CTAACTCGAGCTTTCCCAGATGCTC-3'. The PCR product was inserted into a $\mathrm{pET} 21 \mathrm{a}$ vector, and pET21a-SfSFGH was cloned into the E. coli BL21(DE3) stain. Transformed cells were grown in LB medium containing $100 \mu \mathrm{g} \mathrm{mL}{ }^{-1}$ ampicillin at $37^{\circ} \mathrm{C}$ until the OD600 reached 0.5 . Then, isopropyl- $\beta$-D-1-thiogalactoside (IPTG) was added to a final concentration of $1 \mathrm{mM}$ and the cells were incubated for $3 \mathrm{~h}$ to overexpress SfSFGH. 

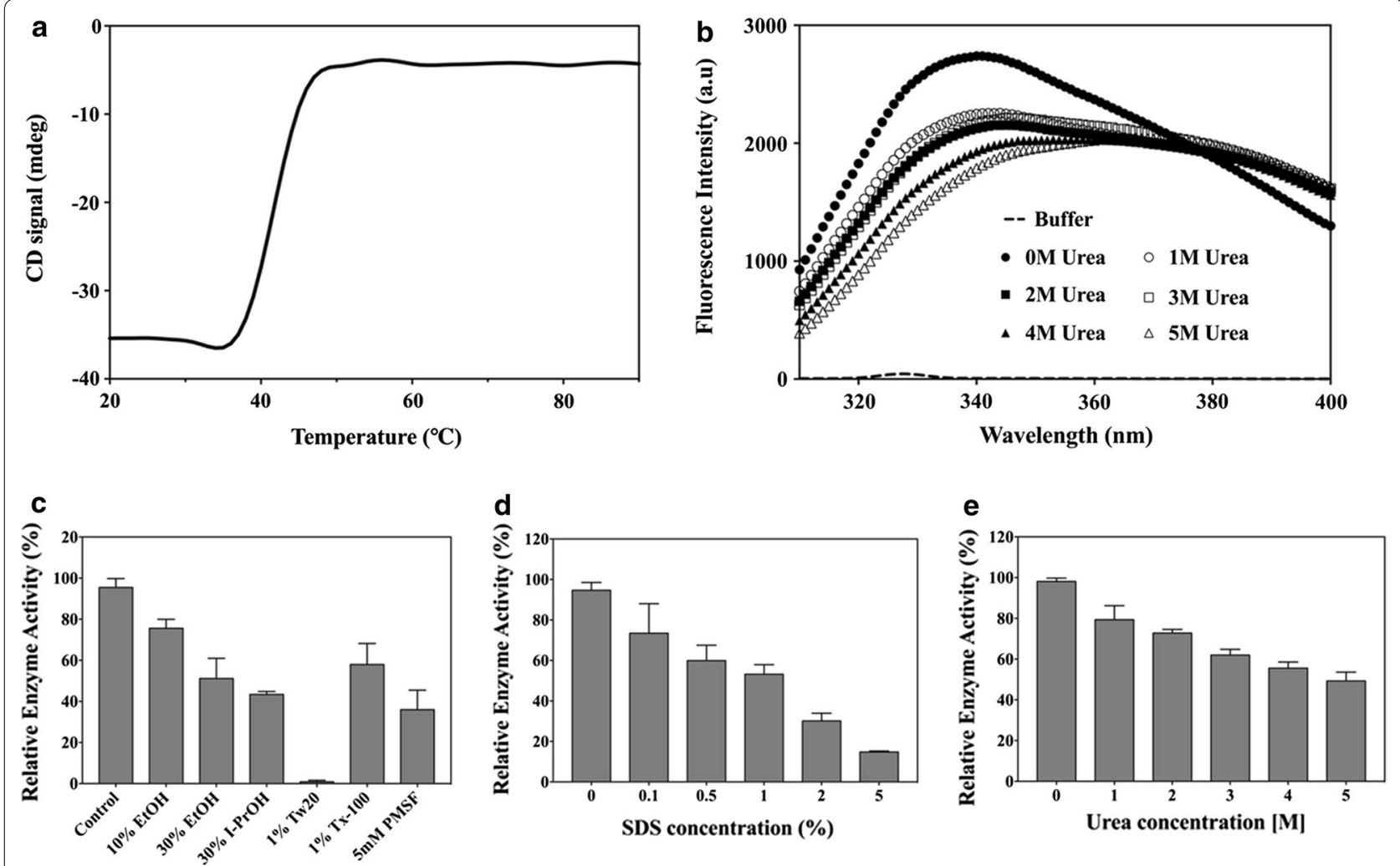

Fig. 6 Stability of SFSFGH. a Thermal unfolding of SfSFGH was monitored at $222 \mathrm{~nm}$ from 20 to $90^{\circ} \mathrm{C}$ using far-UV CD measurements. $\mathbf{b}$ Intrinsic fluorescence spectra were recorded with increasing concentrations of urea from 0 to $5 \mathrm{M}$. c Chemical stability of SfSFGH. Residual activities were measured after incubation of the enzyme for $1 \mathrm{~h}$ in various conditions. Effects of SDS (d) and urea (e) on the enzymatic activity of SfSFGH. All experiments were performed in triplicate

The cells were harvested by centrifugation at $5000 \mathrm{rpm}$ for $20 \mathrm{~min}$ at $4{ }^{\circ} \mathrm{C}$. The resulting pellet was then resuspended in binding buffer $(20 \mathrm{mM}$ Tris $-\mathrm{HCl}, 300 \mathrm{mM}$ $\mathrm{NaCl}, 20 \mathrm{mM}$ imidazole, $\mathrm{pH} 8.0$ ), followed by sonication. The lysate was centrifuged at $40,000 \times g$ for $10 \mathrm{~min}$, and the supernatant was applied to a $1 \mathrm{~mL}$ HisTrap FF column under the AKTA Start system (GE Healthcare). After an extensive washing step with binding buffer, bound SfSFGH was eluted with elution buffer $(20 \mathrm{mM}$ Tris- $\mathrm{HCl}, 300 \mathrm{mM} \mathrm{NaCl}, 250 \mathrm{mM}$ imidazole, $\mathrm{pH}$ 8.0). The purified SfSFGH was desalted using a PD-10 column (GE Healthcare) with an assay buffer $(20 \mathrm{mM}$ Tris- $\mathrm{HCl}$, $200 \mathrm{mM} \mathrm{NaCl}, \mathrm{pH}$ 8.0). The molecular mass of SfSFGH in the native state was estimated using size exclusion chromatography with a Sephacryl S200 HR column (GE healthcare) in an AKTA Start FPLC system (GE healthcare).

\section{Crystallization and data collection of SfSFGH}

Initial crystallization screening of SfSFGH was performed using the sitting-drop vapor-diffusion method at $293 \mathrm{~K}$ in 96-well crystallization plates (Emerald Bio, Bainbridge
Island, WA, USA) using a Mosquito crystallization robot system (TTP Labtech, Cambridge, MA, USA) with commercially available screening kits (MCSG I-IV; Microlytic Burlington, MA, USA). Crystallization drops consisting of $300 \mathrm{~nL}$ protein solution mixed with $300 \mathrm{~nL}$ precipitant solution were equilibrated against $80 \mu \mathrm{L}$ reservoir solution. Crystals of SfSFGH appeared in the condition of $0.2 \mathrm{M}$ lithium chloride and 20\% (w/v) PEG 3350 (MCSG II \#A11). Further optimization of crystallization was conducted in 24-well plates using the hanging-drop vapor-diffusion method by varying the salt and PEG concentrations. Optimized crystals of SfSFGH were obtained from the condition of $0.2 \mathrm{M}$ lithium chloride and $16 \%$ (w/v) PEG 3350. Before mounting, a single crystal of SfSFGH was briefly soaked in Paratone-N oil (Hampton Research, Aliso Viejo, USA) for cryoprotection from the nitrogen-gas stream $(100 \mathrm{~K})$. Diffraction data were collected on a beamline BL-5C at the Pohang Accelerator Laboratory (PAL; Pohang, Korea) using X-rays at a wavelength of $0.9796 \AA$. The crystal was oscillated by $1^{\circ}$ after every $1 \mathrm{~s}$ exposure. Data containing 200 images were 
Table 3 X-ray diffraction data collection and refinement statistics

\begin{tabular}{|c|c|}
\hline Data set & SfSFGH \\
\hline X-ray source & PAL-5C beam line \\
\hline Space group & $P 2_{1}$ \\
\hline Unit-cell parameters $\left(\AA,^{\circ}\right)$ & $\begin{array}{r}a=49.4, b=76.6, c=64.7 \\
a=\gamma=90, \text { and } \beta=105.3\end{array}$ \\
\hline Wavelength ( $\AA$ ) & 0.9796 \\
\hline Resolution ( $\AA$ ) & $50.00-2.32(2.36-2.32)$ \\
\hline Total reflections & 69,131 \\
\hline Unique reflections & $19,691(911)$ \\
\hline Average $\mid / \sigma(I)$ & $42.7(22.4)$ \\
\hline$R_{\text {merge }}^{\mathrm{a}}$ & $0.067(0.165)$ \\
\hline Redundancy & $3.5(3.5)$ \\
\hline Completeness (\%) & $97.0(90.2)$ \\
\hline \multicolumn{2}{|l|}{ Refinement } \\
\hline Resolution range $(\AA)$ & $33.81-2.32(2.38-2.32)$ \\
\hline No. of reflections of working set & $18,706(1341)$ \\
\hline No. of reflections of test set & $964(47)$ \\
\hline No. of amino acid residues & 554 \\
\hline No. of water molecules & 208 \\
\hline$R_{\text {cryst }}^{\mathrm{b}}$ & $0.176(0.165)$ \\
\hline$R_{\text {free }}^{c}$ & $0.235(0.239)$ \\
\hline R.m.s. bond length $(\AA)$ & 0.0096 \\
\hline R.m.s. bond length $\left(^{\circ}\right)$ & 1.596 \\
\hline Average B value $\left(\AA^{2}\right)$ (protein) & 27.9 \\
\hline Average B value $\left(\AA^{2}\right)$ (solvent) & 31.0 \\
\hline
\end{tabular}

Values in parentheses refer to the highest resolution shells

${ }^{\text {a }} R_{\text {merge }}=\sum|\langle I\rangle-\|| / \sum\langle l\rangle$

${ }^{\mathrm{b}} R_{\text {cryst }}=\sum|| \mathrm{Fo}|-| \mathrm{Fc}|| / \sum|\mathrm{Fo}|$

${ }^{c} R_{\text {free }}$ calculated with $5 \%$ of all reflections excluded from the refinement stages using high-resolution data

processed and scaled using $H K L-2000$ [26]. Data collection and processing statistics are shown in Table 3.

\section{Structure determination}

The SfSFGH crystal belongs to the monoclinic space group of $P 2_{1}$, with unit cell parameters of $\mathrm{a}=49.4$, $\mathrm{b}=76.6, \mathrm{c}=64.7 \AA, \alpha=\gamma=90$, and $\beta=105.3^{\circ}$. The crystal structure in SfSFGH was solved by a molecular replacement method using the MOLREP program from the CCP4i package [27, 28]. The crystal structure of esterase (OLEI01171) from Oleispira Antarctica (sequence identity 83\%; PDB code 3i6y) was used as a template model [16]. The initial model was built iteratively and then refined using Coot and REFMAC5 [29, 30]. The phenix.refine from PHENIX was also used for refinement [31]. The final model had an $R_{\text {work }}$ of $17.6 \%$ and $R_{\text {free }}$ of $23.5 \%$. Molprobity was used for quality validation of the final model [32]. The detailed refinement statistics are provided in Table 3. The atomic coordinates and experimental structure factors of SfSFGH were deposited in the RCSB Protein Data Bank under accession code 6JZL. All graphical representations of protein structures were prepared using $P y M O L$ [33].

\section{Mass spectrometry and analytical ultracentrifugation}

The molecular weight of SfSFGH-His ${ }_{6}$ was determined by matrix-assisted laser desorption/ionization time of flight mass spectrometry (MALDI-TOF) using Voyager DE STR (Applied Biosystems, NCIRF, Seoul, Korea). To investigate the oligomeric state of SfSFGH in solution, analytical ultracentrifugation (AUC) was performed using a ProteomeLab XL-A (Beckman Coulter, Brea, CA, USA), in buffer conditions of $150 \mathrm{mM} \mathrm{NaCl}$ and $20 \mathrm{mM}$ Tris- $\mathrm{HCl} \mathrm{pH} 8.0$ at $20{ }^{\circ} \mathrm{C}$. The sample was centrifuged at 40,000 $\mathrm{rpm}$ for $10 \mathrm{~min}$ and the sedimentation profile was monitored at a wavelength of $280 \mathrm{~nm}$. Data were analyzed using the program SEDFIT $[34,35]$.

\section{Enzymatic assays}

In general, the carboxyl esterase activity of SfSFGH was assessed against $p$-nitrophenyl esters by measuring absorbance at $405 \mathrm{~nm}$ using an EPOCH2 microplate reader (Biotek). The standard assay solution consisted of $250 \mu \mathrm{M}$ substrate solution in $100 \mathrm{mM} \mathrm{NaCl}$ and $20 \mathrm{mM}$ Tris $-\mathrm{HCl}$ pH 8.0 with $10 \mu \mathrm{g}$ SfSFGH protein (at a final concentration of $\sim 1.03 \mu \mathrm{M}$ ). The substrate specificity of SfSFGH was determined using $p$-nitrophenyl esters varying in acyl-chain length, e.g., $p$-nitrophenyl phosphate $(p$-NP), $p$-nitrophenyl acetate $(p$-NA), butyrate $(p-\mathrm{NB})$, hexanoate $(p-\mathrm{NH})$, octanoate $(p-\mathrm{NO})$, decanoate $(p-\mathrm{ND})$, and dodecanoate ( $p$-NDD).

The optimum $\mathrm{pH}$ of SfSFGH was determined using $p$-NA with buffers at different $\mathrm{pH}$. Enzymatic activities that differed by $\mathrm{pH}$ were measured after incubating the enzyme for $1 \mathrm{~h}$ at various $\mathrm{pH}$ values ranging from 3.0 to 10.0. The thermal stability of SfSFGH was examined using $p$-NA. Activity changes by temperature were measured after incubating the enzyme at $25{ }^{\circ} \mathrm{C}, 37^{\circ} \mathrm{C}$, $50{ }^{\circ} \mathrm{C}, 60{ }^{\circ} \mathrm{C}$, and $70{ }^{\circ} \mathrm{C}$ for $1 \mathrm{~h}$. Aliquots were taken every $15 \mathrm{~min}$ to measure the residual activity of SfSFGH at each given temperature. To assess effect of low temperature on the activity of SfSFGH, activity was studied by equilibrating the enzyme at $4{ }^{\circ} \mathrm{C}$. The activity was measured using $p$-NA as a substrate. The chemical stability of SfSFGH was determined using $p$-NA with buffers containing diverse chemicals. The residual activities of SfSFGH were measured after incubating the enzyme for $1 \mathrm{~h}$ in diverse conditions, such as $10 \%$ and $30 \%$ ethanol $(\mathrm{EtOH}), 30 \%$ isopropanol ( $\mathrm{I}-\mathrm{PrOH}), 0.1 \%$ sodium dodecyl sulfate (SDS), urea (from $0 \mathrm{M}$ to $5 \mathrm{M}$ ), $1 \%$ 
Triton X-100 (Tx-100), 1\% Tween 20 (Tw20), and $5 \mathrm{mM}$ phenylmethylsulfonyl fluoride (PMSF). Measurement of enzymatic activity was performed in the same manner as the esterase activity assay described above.

\section{Thermal and chemical unfolding}

The thermal unfolding of SfSFGH was assessed using Far-UV CD measurements on a J-715 spectropolarimeter (JASCO) equipped with a thermostat-containing cell holder. The CD signal was monitored at $222 \mathrm{~nm}$ from 20 to $90{ }^{\circ} \mathrm{C}$ at a rate of $1.0^{\circ} \mathrm{C} \mathrm{min}{ }^{-1}$. Chemical unfolding of SfSFGH was induced by incubating the protein with increasing concentrations of urea (from 0 to $5 \mathrm{M}$ ) at room temperature for $1 \mathrm{~h}$. Fluorescence measurements were performed using a FP-6200 spectrofluorometer (JASCO) in which the samples were excited at $280 \mathrm{~nm}$ and emission spectra were recorded in the range of 300 to $400 \mathrm{~nm}$. All fluorescence spectra were recorded at a scan speed of $250 \mathrm{~nm} \mathrm{~min}^{-1}$ with a 5 -nm slit width.

\section{Determination of kinetic parameters}

Kinetic assays were performed using $p$-NA, $p$-NB, and $p$-NO as substrates. Enzyme solutions were prepared in $120 \mathrm{mM} \mathrm{NaCl}$ and $20 \mathrm{mM}$ Tris- $\mathrm{HCl} \mathrm{pH} 8.0$ buffer. Various concentrations of substrate were added to the enzyme solutions, and the reactions were performed at RT. The absorbance changes in the reaction mixtures were monitored using an $\mathrm{EPOCH} 2$ microplate reader (Biotek) at $405 \mathrm{~nm}$. Measurements were taken along with a blank, every $10 \mathrm{~s}$ for $2 \mathrm{~min}$, and at least three measurements were collected to determine the rate at each substrate concentration. The molar extinction coefficients of $p$-nitrophenol and hydrolyzed nitrocefin were determined experimentally ( $p$-nitrophenol; 17,154 $\mathrm{M}^{-1} \mathrm{~cm}^{-1}$ ). Reaction velocities were determined from the initial linear portion of progress curves, which were generated after subtracting the absorbances of the blank experiments, correcting sample path-length, and converting the absorbance to product concentration $[\mathrm{P}]$ with the experimentally obtained extinction coefficient. The kinetic parameters $\left(V_{\max }\right.$ and $K_{\mathrm{m}}$ ) of SfSFGH were obtained from the $\mathrm{x}$ - and $\mathrm{y}$-intercepts of the Lineweaver-Burk plot. The $k_{\text {cat }}$ was calculated using the equation $k_{\mathrm{cat}}=V_{\max } /[\mathrm{E}]_{\text {total }}$.

\section{Bioinformatics analysis}

For functional classification and sequence analysis of SfSFGH, proteins related to SfSFGH were identified using a BLAST search on the Protein Data Bank (PDB). All primary sequences were retrieved from the NCBI database in FASTA format. Multiple sequence alignments were performed using Clustal Omega [36] with the enzymes found using BLAST, and the results were rendered using
ESPript [37]. For gene cluster analysis, homologous proteins of SfSFGH (sequence identity: $\geq 80 \%$ ) were collected from a BLAST search on the non-redundant protein sequence database, followed by retrieval of chromosome information for the collected proteins. The gene locus encoding SfSFGH and its surroundings in the chromosome of Shewanella frigidimarina were compared with the corresponding regions in other Shewanella species, including S. sediminis, S. woodyi, S. pealeana, and S. piezotolerans.

\section{Site-directed mutagenesis}

To obtain mutants of SfSFGH, mutagenesis was carried out using the Quik-Change site-directed mutagenesis method [19]. Chemically synthesized oligonucleotides were used to substitute each residue (purchased from Cosmogenetech, Korea). In the Quik-Change method, the PCR mixture contained $\sim 300$ ng DNA template, $4 \mu \mathrm{L} 5 \times$ Pfu DNA polymerase master mix, and $1 \mu \mathrm{M}$ of each primer in a total volume of $20 \mu \mathrm{L}$. The mixture was heated at $95^{\circ} \mathrm{C}$ for 2 min and then subjected to thermal cycling ( 18 cycles at $95^{\circ} \mathrm{C}$ for $1 \mathrm{~min}, 66^{\circ} \mathrm{C}$ for $1 \mathrm{~min}$, and $72{ }^{\circ} \mathrm{C}$ for $7 \mathrm{~min}$ ). The PCR product was incubated with DpnI at $37^{\circ} \mathrm{C}$ for $1 \mathrm{~h}$, subsequently incubated for $10 \mathrm{~min}$ at $80{ }^{\circ} \mathrm{C}$, and transformed into E. coli $\mathrm{DH} 5 \alpha$. Three colonies were randomly selected for sequencing. The verified plasmid was then transformed into E. coli BL21(DE3) for overexpression.

\section{Additional file}

Additional file 1: Fig. S1. Phylogenetic analysis of SFSFGH. Fig. S2. Gene clustering analysis of SFSFGH. Fig. S3. Recombinant SFSFGH protein purification, crystallization, and X-ray diffraction data collection. Fig. S4. Freeze-thaw cycles of SFSFGH.

\section{Acknowledgements}

We thank the staff at the X-ray core facility of the Korea Basic Science Institute (Ochang, Korea) and BL-5C of the Pohang Accelerator Laboratory (Pohang, Korea) for providing assistance with data collection. TDK would like to thank Jinhee Kim for helping with this study.

\section{Authors' contributions}

TDK and JHL designed and supervised the project. CWL, WY, S-HP, LTHLL, and CSJ performed cloning, expression, and protein purification. CWL and $\mathrm{S}$-HP crystallized the protein, collected $\mathrm{X}$-ray diffraction data, and solved the protein structure. S-HP, BHR, H-WK, SCS, HP, and TDK performed biochemical characterization experiments. CWL, WY, S-HP, TDK, and JHL wrote the initial manuscript, and HP contributed to revisions. All authors discussed the results, commented on the manuscript. All authors read and approved the final manuscript.

\section{Funding}

This work was supported by the Korea Polar Research Institute (Grant Number PE19210 to JHL). This work was also supported by the National Research Foundation of Korea (NRF) funded by the Ministry of Science, ICT and Future 
Planning (MSIP) [application study on Arctic cold-active enzymes degrading organic carbon compounds; NRF Grant Number NRF-2017M1A5A1013568 and KOPRI Grant Number PN19082 to H-WK]. This work was also supported by a grant from the National Research Foundation of Korea funded by the Korean Government (NRF-2017M1A5A1013569 and NRF-2018R1D1A1B070444477 to TDK).

\section{Ethics approval and consent to participate}

Not applicable.

\section{Consent for publication}

Not applicable.

\section{Competing interests}

The authors declare that they have no competing interests.

\section{Author details}

${ }^{1}$ Unit of Polar Genomics, Korea Polar Research Institute, Incheon 21990, Republic of Korea. ${ }^{2}$ Department of Chemistry, College of Natural Science, Sookmyung Woman's University, Seoul 04310, Republic of Korea. ${ }^{3}$ Department of Molecular Cell Biology, Samsung Biomedical Research Institute, Sungkyunkwan University School of Medicine, Suwon 16419, Republic of Korea. ${ }^{4}$ Department of Polar Sciences, University of Science and Technology, Incheon 21990, Republic of Korea. ${ }^{5}$ Division of Biotechnology, College of Life Sciences and Biotechnology, Korea University, Seoul 02841, Republic of Korea. ${ }^{6}$ Present Address: Public CDMO for Microbial-based Vaccine, Infrastructure Project Organization for Global Industrialization of Vaccine, Hwasun 58141, Republic of Korea.

Received: 4 June 2019 Accepted: 8 August 2019 Published online: 19 August 2019

\section{References}

1. Berg J, Tymoczko J, Stryer L. 9.1 Proteases: facilitating a difficult reaction. Biochemistry. 5th ed. New York: WH Freeman; 2002.

2. Sun Y, Yin S, Feng Y, Li J, Zhou J, Liu C, et al. Molecular basis of the general base catalysis of an alpha/beta-hydrolase catalytic triad. J Biol Chem. 2014;289:15867-79

3. Rauwerdink A, Kazlauskas RJ. How the same core catalytic machinery catalyzes 17 different reactions: the serine-histidine-aspartate catalytic triad of alpha/beta-hydrolase fold enzymes. ACS Catal. 2015;5:6153-76.

4. Herring CD, Blattner FR. Global transcriptional effects of a suppressor tRNA and the inactivation of the regulator frmR. J Bacteriol. 2004:186:6714-20.

5. Gonzalez CF, Proudfoot M, Brown G, Korniyenko Y, Mori H, Savchenko AV, et al. Molecular basis of formaldehyde detoxification. Characterization of two S-formylglutathione hydrolases from Escherichia coli, FrmB and YeiG. J Biol Chem. 2006;281:14514-22.

6. Harms N, Ras J, Reijnders WN, van Spanning RJ, Stouthamer AH. S-formylglutathione hydrolase of Paracoccus denitrificans is homologous to human esterase D: a universal pathway for formaldehyde detoxification? J Bacteriol. 1996; 178:6296-9.

7. Lee WH, Wheatley W, Benedict WF, Huang CM, Lee EY. Purification, biochemical characterization, and biological function of human esterase D. Proc Natl Acad Sci USA. 1986;83:6790-4.

8. Wu D, LiY, Song G, Zhang D, Shaw N, Liu ZJ. Crystal structure of human esterase D: a potential genetic marker of retinoblastoma. FASEB J. 2009;23:1441-6.

9. Lee EY, Lee WH. Molecular cloning of the human esterase $D$ gene, a genetic marker of retinoblastoma. Proc Natl Acad Sci USA. 1986;83:6337-41.

10. Kordic S, Cummins I, Edwards R. Cloning and characterization of an $S$-formylglutathione hydrolase from Arabidopsis thaliana. Arch Biochem Biophys. 2002;399:232-8.

11. Cummins I, McAuley K, Fordham-Skelton A, Schwoerer R, Steel PG, Davis $B G$, et al. Unique regulation of the active site of the serine esterase S-formylglutathione hydrolase. J Mol Biol. 2006;359:422-32.

12. Uotila L, Koivusalo M. Purification of formaldehyde and formate dehydrogenases from pea seeds by affinity chromatography and
S-formylglutathione as the intermediate of formaldehyde metabolism. Arch Biochem Biophys. 1979;196:33-45.

13. Degrassi G, Uotila L, Klima R, Venturi V. Purification and properties of an esterase from the yeast Saccharomyces cerevisiae and identification of the encoding gene. Appl Environ Microbiol. 1999;65:3470-2.

14. Yurimoto H, Lee B, Yano T, Sakai Y, Kato N. Physiological role of S-formylglutathione hydrolase in C(1) metabolism of the methylotrophic yeast Candida boidinii. Microbiology. 2003;149:1971-9.

15. van Straaten KE, Gonzalez CF, Valladares RB, Xu X, Savchenko AV, Sanders DA. The structure of a putative $S$-formylglutathione hydrolase from Agrobacterium tumefaciens. Protein Sci. 2009;18:2196-202.

16. Lemak S, Tchigvintsev A, Petit P, Flick R, Singer AU, Brown G, et al. Structure and activity of the cold-active and anion-activated carboxyl esterase OLEI01171 from the oil-degrading marine bacterium Oleispira antarctica. Biochem J. 2012;445:193-203.

17. Aurilia V, Parracino A, Saviano M, Rossi M, D'Auria S. The psychrophilic bacterium Pseudoalteromonas halosplanktis TAC125 possesses a gene coding for a cold-adapted feruloyl esterase activity that shares homology with esterase enzymes from gamma-proteobacteria and yeast. Gene. 2007:397:51-7.

18. Alterio V, Aurilia V, Romanelli A, Parracino A, Saviano M, D'Auria S, et al. Crystal structure of an S-formylglutathione hydrolase from Pseudoalteromonas haloplanktis TAC125. Biopolymers. 2010;93:669-77.

19. Li S, Wilkinson MF. Site-directed mutagenesis: a two-step method using PCR and Dpnl. Biotechniques. 1997;23:588-90.

20. Gasteiger E, Hoogland C, Gattiker A, Wilkins MR, Appel RD, Bairoch A. Protein identification and analysis tools on the ExPASy server. The proteomics protocols handbook. Berlin: Springer; 2005. p. 571-607.

21. Krauss U, Jager VD, Diener M, Pohl M, Jaeger KE. Catalytically-active inclusion bodies-carrier-free protein immobilizates for application in biotechnology and biomedicine. J Biotechnol. 2017;258:136-47.

22. Sheldon RA. Cross-linked enzyme aggregates (CLEAs): stable and recyclable biocatalysts. Biochem Soc Trans. 2007;35:1583-7.

23. Holm L, Rosenstrom P. Dali server: conservation mapping in 3D. Nucleic Acids Res. 2010;38:W545-9.

24. Chen NH, Counago RM, Djoko KY, Jennings MP, Apicella MA, Kobe $B$, McEwan AG. A glutathione-dependent detoxification system is required for formaldehyde resistance and optimal survival of Neisseria meningitidis in biofilms. Antioxid Redox Signal. 2013;18:743-55.

25. Legler PM, Kumaran D, Swaminathan S, Studier FW, Millard CB. Structural characterization and reversal of the natural organophosphate resistance of a D-type esterase, Saccharomyces cerevisiae S-formylglutathione hydrolase. Biochemistry. 2008;47:9592-601.

26. Otwinowski Z, Minor W. Processing of X-ray diffraction data collected in oscillation mode. Methods Enzymol. 1997;276:307-26.

27. Vagin A, Teplyakov A. Molecular replacement with MOLREP. Acta Crystallogr D Biol Crystallogr. 2010;66:22-5.

28. Winn MD, Ballard CC, Cowtan KD, Dodson EJ, Emsley P, Evans PR, Keegan RM, Krissinel EB, Leslie AG, McCoy A, et al. Overview of the CCP4 suite and current developments. Acta Crystallogr D Biol Crystallogr. 2011;67:235-42.

29. Emsley P, Cowtan K. Coot: model-building tools for molecular graphics. Acta Crystallogr D Biol Crystallogr. 2004;60:2126-32.

30. Murshudov GN, Skubak P, Lebedev AA, Pannu NS, Steiner RA, Nicholls RA, Winn MD, Long F, Vagin AA. REFMAC5 for the refinement of macromolecular crystal structures. Acta Crystallogr D Biol Crystallogr. 2011:67:355-67.

31. Adams PD, Afonine PV, Bunkoczi G, Chen VB, Davis IW, Echols N, Headd JJ, Hung LW, Kapral GJ, Grosse-Kunstleve RW, et al. PHENIX: a comprehensive Python-based system for macromolecular structure solution. Acta Crystallogr D Biol Crystallogr. 2010;66:213-21.

32. Chen VB, Arendall WB 3rd, Headd JJ, Keedy DA, Immormino RM, Kapral GJ, Murray LW, Richardson JS, Richardson DC. MolProbity: all-atom structure validation for macromolecular crystallography. Acta Crystallogr D Biol Crystallogr. 2010;66:12-21.

33. DeLano WL. Pymol: an open-source molecular graphics tool. CCP4 Newsl Protein Crystallogr. 2002;40:82-92.

34. Schuck P. Size-distribution analysis of macromolecules by sedimentation velocity ultracentrifugation and lamm equation modeling. Biophys J. 2000;78:1606-19. 
35. Schuck P, Rossmanith P. Determination of the sedimentation coefficient distribution by least-squares boundary modeling. Biopolymers. 2000;54:328-41.

36. Sievers F, Wilm A, Dineen D, Gibson TJ, Karplus K, Li W, Lopez R, McWilliam H, Remmert M, Soding J, et al. Fast, scalable generation of highquality protein multiple sequence alignments using Clustal Omega. Mol Syst Biol. 2011;7:539.

37. Gouet P, Robert X, Courcelle E. ESPript/ENDscript: extracting and rendering sequence and $3 \mathrm{D}$ information from atomic structures of proteins. Nucleic Acids Res. 2003;31:3320-3.
38. Legler PM, Leary DH, Hervey WJT, Millard CB. A role for His-160 in peroxide inhibition of $S$. cerevisiae S-formylglutathione hydrolase: evidence for an oxidation sensitive motif. Arch Biochem Biophys. 2012;528:7-20.

\section{Publisher's Note}

Springer Nature remains neutral with regard to jurisdictional claims in published maps and institutional affiliations.
Ready to submit your research? Choose BMC and benefit from:

- fast, convenient online submission

- thorough peer review by experienced researchers in your field

- rapid publication on acceptance

- support for research data, including large and complex data types

- gold Open Access which fosters wider collaboration and increased citations

- maximum visibility for your research: over $100 \mathrm{M}$ website views per year

At BMC, research is always in progress.

Learn more biomedcentral.com/submissions 